\title{
Adaptabilidade climática de caprinos Saanen e Azul no Meio-Norte do Brasil
}

\author{
[Climatic adaptability of Saanen and Azul goats in Brazilian Middle-North] \\ R.R.C. Rocha ${ }^{1}$, A.P.R. Costa ${ }^{2}$, D.M.M.R. Azevedo ${ }^{3 *}$, H.T.S. Nascimento ${ }^{4}$, F.S. Cardoso ${ }^{2}$, \\ M.C.S. Muratori ${ }^{3}$, J.B. Lopes $^{3}$ \\ ${ }^{1}$ Aluno de pós-graduação - CCA-UFPI - Teresina, PI \\ ${ }^{2}$ Centro de ciências Agrárias - UFPI - Teresina, PI \\ ${ }^{3}$ Embrapa Meio-Norte-UEP/Parnaíba \\ BR 343, Km 35, \\ 64200-970 - Parnaíba, PI \\ ${ }^{4}$ Embrapa Meio-Norte - Teresina, PI
}

\begin{abstract}
RESUMO
A adaptabilidade de caprinos de dois grupos genéticos, Saanen e Azul, às condições climáticas do MeioNorte do Brasil foi avaliada por meio dos testes de Ibéria, Benezra e Rainsby. Nos dois primeiros, foram utilizadas sete fêmeas de cada grupo racial e, no terceiro, quatro fêmeas de cada grupo. Foram realizadas quatro coletas de dados em cada período (chuvoso e seco) de 2005. Foi utilizado delineamento inteiramente ao acaso, em fatorial $2 \times 2$ (duas raças e dois períodos). Os valores do coeficiente de tolerância ao calor do teste de Ibéria no período seco diferiram entre os grupos $(\mathrm{P}<0,05)$ (Saanen $=97,65$ e Azul $=94,31)$. Houve diferença entre grupos $(P<0,05)$ quanto ao coeficiente de adaptabilidade 1 do teste de Benezra, nos dois períodos (chuvoso: Saanen $=5,13$ e Azul =3,26; seco: Saanen $=5,86$ e Azul = 2,87 ). No teste de Rainsby, no grupo Azul, houve o retorno à temperatura de repouso nos dois períodos. $\mathrm{Na}$ Saanen, no período no seco, 100 minutos não foram suficientes para o retorno à temperatura de repouso. O grupo racial Azul mostrou-se mais adaptado às condições do Meio-Norte.
\end{abstract}

Palavras-chave: caprino, adaptação, climática, estresse térmico, índices

\begin{abstract}
This study was undertaken to evaluate Saanen and Azul goats' adaptability to the Brazilian Middle-North region, based on adaptation indexes (Iberia, Benezra and Rainsby tests). A totall of seven and four females, respectively, of each group were used in two tests and four collections were performed during the rainy and dry periods of 2005 year. A completely randomized experimental design in a $2 \times 2$ (2 groups $x 2$ period) factorial treatment combination was used. Significant difference between groups (Saanen $=97.65$ and Azul $=94.31)$ was observed for heat-tolerance coefficient (Iberia) during the dry period $(P<.05)$. Significant differences between groups $(P<0.05)$ were also observed for adaptability, coefficient 1 (Benezra) for both rainy (Saanen $=5.13$ and Azul $=3.26$ ) and dry periods (Saanen $=5.86$ and Azul $=2.87)$. Based on Rainsby test, Azul goats returned to rest temperature in both periods. During the dry period 100 minutes were not enough for Saanen goats return to rest temperature. Azul goats showed higher adaptability to environment conditions of the Brazilian Middle-North region.
\end{abstract}

Keywords: adaptation, bioclimatology, heat stress, indexes

Recebido em 06 de maio de 2009

Aceito em 20 de outubro de 2009

*Autor para correspondência (corresponding author)

E-mail: azevedo@cpamn.embrapa.br

Pesquisa financiada pelo MCT/CNPq/PADCT. Projeto Casadinho. Região Nordeste. 


\section{INTRODUÇÃO}

$\mathrm{Na}$ região Nordeste predomina o sistema extensivo de criação de caprinos, que predispõe os animais a condições de temperatura e umidade inadequadas em determinadas épocas do ano.

Considerando que a produtividade ou mesmo a sobrevivência do animal depende, principalmente, de sua capacidade em manter a temperatura corporal dentro de certos limites, independente das variações de temperatura do ambiente (homeotermia) (Johnson, 1987), pesquisas referentes às condições climáticas associadas com estudos de parâmetros fisiológicos, como temperatura retal e frequências cardíaca e respiratória (Ferreira et al., 2006; Silva et al., 2007; Morais et al., 2008; Maia et al., 2009). Essas pesquisas são, também, importantes para se conhecer a adaptabilidade dos animais criados em determinada região (Souza et al., 2007), servindo ainda para comparar diferentes grupos genéticos (Santos et al., 2003; Neiva et al., 2004; Turco et al., 2004; Martins Júnior et al., 2007b; Souza et al., 2008a, b).

Além destes parâmetros fisiológicos, testes ou provas de adaptação ou de tolerância ao calor têm sido realizados com o intuito de facilitar a mensuração da adaptabilidade de determinado animal ou grupo genético, por meio de sua capacidade em manter sua homeotermia. Estes testes são utilizados, principalmente, para bovinos (Magalhães et al., 2000), pois foram desenvolvidos, originalmente, para essa espécie (Muller, 1982), sendo, ainda, pouco utilizados para caprinos (Martins Júnior et al., 2007a; Souza et al., 2008a, b) e equinos (Oliveira et al., 2008).

No Nordeste do Brasil, existem diversos grupos genéticos de caprinos nativos, produtos da seleção natural para o ambiente climático da região. Entre os nativos, encontra-se o grupo racial Azul, de pelagem cinza azulada e pele escura, ainda não reconhecido como raça. São encontrados exemplares isolados de caprinos Azul em rebanhos de várias partes do Nordeste. No Estado do Piauí, é raro encontrar essa variedade. A Fazenda Cajaíba, no município de Teresina, mantém, há vários anos, um plantel preservado, com apoio da Embrapa Meio-Norte (Associação..., 2005). Além dos animais nativos, animais exóticos, oriundos, principalmente, da África e Europa foram introduzidos na Região Nordeste, como é o caso da raça suíça Saanen, especializada em produção de leite. Animais dessa raça apresentam pelagem branca, pelos curtos e finos, pele rosada e aberturas naturais amarelas (Jardim, 1987).

O objetivo deste trabalho foi avaliar a adaptabilidade do grupo racial Azul e da raça Saanen, por meio da aplicação de testes de adaptabilidade às condições climáticas da Região Meio-Norte do Brasil.

\section{MATERIAL E MÉTODOS}

Foram realizados três testes distintos: Ibéria ou Rhoad, Benezra e Rainsby, adaptados para caprinos, todos aplicados na Fazenda Cajaíba, distante $25 \mathrm{~km}$ de Teresina, PI, utilizando-se caprinos Saanen e Azul, fêmeas, jovens, não prenhes, em bom estado clínico e nutricional, com peso médio de 35,6 e $28,3 \mathrm{~kg}$, respectivamente.

O teste de Ibéria ou Rhoad, realizado para determinação do coeficiente de tolerância ao calor (CTC), utilizou sete animais de cada grupo racial, escolhidos ao acaso, que ficaram expostos ao sol durante 30 minutos, em dois horários distintos, 10 e 15 horas, com a temperatura ambiente entre 29,5 e $35^{\circ} \mathrm{C}$ (Muller, 1982).

As coletas de dados foram realizadas uma vez a cada 15 dias, durante dois meses, nos períodos chuvoso (janeiro/fevereiro) e seco (setembro/outubro) de 2005.

A fórmula utilizada para determinar o CTC foi: $\mathrm{CTC}=100-[18 \quad(\mathrm{TR}-39,1)]$, em que $\mathrm{CTC}=$ coeficiente de tolerância ao calor; $100=$ eficiência máxima em manter a temperatura corporal em $39,1^{\circ} \mathrm{C} ; 18=$ constante; $\mathrm{TR}=$ temperatura retal média final; $39,1^{\circ} \mathrm{C}=$ temperatura retal média considerada normal para caprinos.

Os mesmos animais foram, também, submetidos ao teste de Benezra, para determinação do coeficiente de adaptabilidade 1 (CA1) (Muller, 1982). Esse teste foi realizado sempre entre $14 \mathrm{e}$ 15 horas, uma vez a cada 15 dias, quatro vezes em cada período (seco e chuvoso). Todos os animais foram mantidos à sombra, em um dia ensolarado com a temperatura ambiente (TA) 
variando de 29,5 a $36^{\circ} \mathrm{C}$. O CA1 foi determinado pela fórmula: $\mathrm{CA} 1=\mathrm{TR} / 39,1+\mathrm{FR} / 19$, em que CA1 = coeficiente de adaptabilidade do teste de Benezra; $\mathrm{TR}=$ temperatura retal, em ${ }^{\circ} \mathrm{C}$; $\mathrm{FR}=$ frequência respiratória, em movimentos por minuto; 39,1 = temperatura retal considerada normal para caprinos; $19=$ frequência respiratória considerada normal para caprinos. $\mathrm{O}$ resultado obtido foi comparado a 2, valor em que os parâmetros fisiológicos utilizados na fórmula não se alteram em relação ao normal.

Visando aumentar a capacidade de detecção do teste, acrescentou-se à formula anterior a frequência cardíaca (FC) e obteve-se o coeficiente de adaptabilidade 2 (CA2 = TR/39, 1 $+\mathrm{FR} / 19+\mathrm{FC} / 75)$. Para caprinos, considerou-se normal a FC de 75 batimentos/minuto, utilizada na fórmula para CA2. O novo coeficiente foi então comparado ao valor 3 .

O teste de Rainsby (Muller, 1982) consistiu em recolher ao aprisco quatro animais de cada grupo genético, escolhidos ao acaso, na noite anterior ao teste para tomada da TR às sete horas do dia seguinte. Após a primeira mensuração, os animais foram submetidos a exercício físico intenso (corrida), durante 10 minutos, para determinação da nova TR. Se a TR de pelo menos um animal não atingisse $40^{\circ} \mathrm{C}$ ou mais, todos eram submetidos a mais 10 minutos de exercícios físicos até que a TR de todos fosse $40^{\circ} \mathrm{C}$ ou superior.

Após atingir $40^{\circ} \mathrm{C}$, realizaram-se novas tomadas da TR a cada 20 minutos, até 100 minutos. O tempo necessário para o retorno à temperatura inicial representou a capacidade de os animais dissiparem o calor produzido durante o exercício físico. Esse teste foi repetido quatro vezes em cada período avaliado.

Utilizando-se a mesma metodologia e animais do teste de Rainsby, mediu-se a FR com a finalidade de confirmar os resultados obtidos para o teste quanto à adaptabilidade das duas raças, verificando-se o tempo necessário para a FR retornar ao valor inicial de repouso pré-exercício.

Durante a realização dos testes, foram obtidas a temperatura ambiente (TA) e a umidade relativa do ar (UR), com o auxílio de termo-higrômetro, e a temperatura de globo negro (TGN), usandose globo-termômetro, instalados à altura de $55 \mathrm{~cm}$ do solo, que corresponde à altura média dos animais. A partir da TA, UR e TGN foram calculados o índice de temperatura e umidade (ITU) e o índice de temperatura de globo e umidade (ITGU): ITU $=0,8 \mathrm{Tbs}+\mathrm{UR}(\mathrm{Tbs}-$ 14,3)/100+46,3, em que Tbs = temperatura do bulbo seco $\left({ }^{\circ} \mathrm{C}\right)$ e UR $=$ umidade relativa do ar e ITGU $=\mathrm{T}_{\mathrm{g}}+$ 0,36Tpo $+41,5$, em que $\mathrm{Tg}=$ temperatura do globo negro $\left({ }^{\circ} \mathrm{C}\right)$, e Tpo = temperatura do ponto de orvalho (\%) (Buffington et al. 1981).

$\mathrm{O}$ delineamento utilizado nos três testes foi $\mathrm{o}$ inteiramente ao acaso, em esquema fatorial $2 \times 2$ (duas raças $\mathrm{x}$ dois períodos), com sete repetições para os testes de Ibéria e Benezra e quatro repetições para o teste de Rainsby. Para diferenciação entre médias, foram utilizados os testes Duncan para o Ibéria e teste SNK, para Benezra e Rainsby, ambos a 5\% de probabilidade. As análises foram realizadas utilizando-se o logiciário estatístico SAS/1997.

\section{RESULTADOS E DISCUSSÃO}

Os resultados do CTC (Tab. 1) mostram que, no período chuvoso, os dois grupos genéticos comportaram-se de maneira similar, porém, no período seco, a raça Saanen foi mais tolerante à exposição ao sol $(\mathrm{P}<0,05)$, pois seu CTC esteve mais próximo de 100 . O melhor desempenho dos Saanen, neste teste, deve-se provavelmente à sua pelagem branca, com alto poder de reflexão dos raios solares. Comportamento semelhante foi observado por Pant et al. (1985), ao verificarem que caprinos nativos de cor branca tiveram menor aumento de TR em relação aos de cor negra, quando expostos ao sol, indicando menor esforço dos primeiros em manter sua homeotermia.

Medeiros et al. (2002), ao aplicarem a cabras de três raças distintas outro teste de exposição ao sol, observaram que a raça Saanen, apesar da pelagem branca, apresentou pior desempenho que a Anglo-Nubiana e comportamento próximo ao da Parda Alemã, ambas de pelagem marrom, denotando maior influência da origem da raça do que da cor da pelagem. Este resultado é aparentemente contraditório ao do teste de Ibéria, porém deve ser considerado que a avaliação dos animais Saanen e Azul foi realizada com UR mais baixa que a do experimento citado por Medeiros et al. (2002), o que pode ter sido determinante para o desempenho do animal. 
Tabela 1. Coeficiente de tolerância ao calor, segundo o teste Ibéria, para caprinos Saanen e Azul, nos períodos chuvoso e seco, no município de Teresina, PI

\begin{tabular}{lcc}
\multicolumn{1}{r}{ Período } & Saanen & Azul \\
\hline Chuvoso & $95,47 \pm 5,97 \mathrm{Aa}$ & $93,70 \pm 4,43 \mathrm{Aa}$ \\
Seco & $97,65 \pm 3,45 \mathrm{Aa}$ & $94,31 \pm 6,49 \mathrm{Ba}$ \\
\hline Média geral & $96,56 \pm 4,95 \mathrm{~A}$ & $94,00 \pm 5,52 \mathrm{~B}$ \\
\hline CV 5,49\% & & \\
\hline
\end{tabular}

Médias na mesma linha seguidas de letras maiúsculas distintas diferem entre si $(\mathrm{P}<0,05)$ pelo teste de Duncan.

Médias na mesma coluna seguidas de letras minúsculas distintas diferem entre si $(\mathrm{P}<0,05)$ pelo teste Duncan.

Martins Júnior et al. (2007a, b), ao estudarem as raças Anglo-Nubiana e Boer na região MeioNorte do Brasil nos períodos seco e chuvoso, por meio do teste de Ibéria, não observaram diferenças $(\mathrm{P}>0,05)$ entre as raças no período chuvoso, porém constataram melhor adaptação da raça Boer $(\mathrm{P}<0,05)$ no período seco.

O CA1 do grupo racial Azul foi mais próximo do ideal que o da raça Saanen (Tab. 2), indicando maior adaptação ao clima quente, tanto no período chuvoso quanto no seco. Em condições climáticas semelhantes, Martins Júnior et al. (2007a) verificaram maior adaptabilidade da raça Anglo-Nubiana no período chuvoso e da Boer no período seco, indicando maior tolerância dos animais Boer a temperaturas mais elevadas, quando associadas a umidades mais baixas.

Tabela 2. Coeficiente de adaptabilidade 1 segundo o teste Benezra, para caprinos Saanen e Azul, nos períodos chuvoso e seco, no município de Teresina, PI

\begin{tabular}{lcc}
\hline Período & Saanen & Azul \\
\hline Chuvoso & $5,13 \pm 1,54 \mathrm{Ab}$ & $3,26 \pm 1,25 \mathrm{Ba}$ \\
Seco & $5,86 \pm 1,39 \mathrm{Aa}$ & $2,87 \pm 0,95 \mathrm{Bb}$ \\
\hline Média & $5,49 \pm 1,51 \mathrm{~A}$ & $3,06 \pm 1,12 \mathrm{~B}$ \\
\hline
\end{tabular}

CV 28,09\%

Médias na mesma linha seguidas de letras maiúsculas distintas diferem entre si $(\mathrm{P}<0,05)$ pelo teste SNK.

Médias na mesma coluna seguidas de letras minúsculas distintas diferem entre si $(\mathrm{P}<0,05)$ pelo teste de SNK.

Quanto ao CA2, não se verificou alteração do resultado final (Tab. 3), confirmando a maior adaptabilidade do grupo racial Azul às condições em que o teste foi realizado. Martins Júnior et al. (2007a) verificaram maior adaptabilidade da raça Boer, no período seco, que a Anglo-Nubiana, porém não encontraram diferença $(\mathrm{P}>0,05)$ entre as duas raças no período chuvoso. Talvez isso se deva à origem africana da Boer, enquanto a Anglo-Nubiana, embora de tronco africano, foi submetida à seleção na Europa.

Tabela 3. Coeficiente de adaptabilidade 2, segundo adaptação do teste Benezra para caprinos Saanen e Azul, nos períodos chuvoso e seco, no município de Teresina, PI

\begin{tabular}{lcc}
\hline Período & Saanen & Azul \\
\hline Chuvoso & $6,19 \pm 1,64 \mathrm{Ab}$ & $4,29 \pm 1,40 \mathrm{Ba}$ \\
Seco & $6,83 \pm 1,41 \mathrm{Aa}$ & $3,77 \pm 0,99 \mathrm{Bb}$ \\
\hline Média & $6,51 \pm 1,56 \mathrm{~A}$ & $4,03 \pm 1,24 \mathrm{~B}$ \\
\hline CV 24,29\% & &
\end{tabular}

Médias na mesma linha seguidas de letras maiusculas distintas diferem entre si $(\mathrm{P}<0,05)$ pelo teste de SNK Médias na mesma coluna seguida de letras maiúsculas distintas diferem entre si $(\mathrm{P}<0,05)$ pelo teste de SNK.

Na raça Saanen, no teste de Rainsby realizado no período chuvoso, a TR retornou ao valor de repouso 40 minutos após o exercício, e não o fez até os 100 minutos, no período seco (Tab. 4). No grupo racial Azul, o retorno à TR de repouso ocorreu aos 20 min no período chuvoso e aos 40min no período seco. Isso mostra a maior rapidez do grupo Azul em dissipar calor. 
Tabela 4. Médias para temperatura retal em acompanhamento ao teste Rainsby para caprinos Saanen e Azul, nos períodos chuvoso e seco, no município de Teresina, PI

\begin{tabular}{lccccc}
\hline \multirow{2}{*}{$\begin{array}{c}\text { Situação/ } \\
\text { Tempo }\end{array}$} & \multicolumn{2}{c}{ Chuvoso } & \multicolumn{2}{c}{ Seco } & CV \\
\cline { 2 - 5 } & Saanen & Azul & Saanen & Azul & $(\%)$ \\
\hline Repouso & $38,87 \pm 0,27 \mathrm{Ab}^{2}$ & $39,19 \pm 0,29 \mathrm{Aa}^{2}$ & $38,51 \pm 0,32 \mathrm{Bb}^{2}$ & $38,87 \pm 0,47 \mathrm{Ba}^{2}$ & 0,78 \\
\hline Exercitado & $40,56 \pm 0,48 \mathrm{Ab}^{1}$ & $40,89 \pm 0,32 \mathrm{Aa}^{1}$ & $40,26 \pm 0,29 \mathrm{Ba}^{1}$ & $40,44 \pm 0,39 \mathrm{Ba}^{1}$ & 0,82 \\
\hline TR20* & $39,71 \pm 0,35 \mathrm{Aa}^{1}$ & $39,26 \pm 1,35 \mathrm{Aa}^{2}$ & $39,41 \pm 0,21 \mathrm{Ba}^{1}$ & $39,34 \pm 0,49 \mathrm{Aa}^{1}$ & 1,85 \\
\hline TR40* & $39,33 \pm 0,26 \mathrm{Aa}^{2}$ & $39,23 \pm 0,33 \mathrm{Aa}^{2}$ & $38,96 \pm 0,34 \mathrm{Ba}^{1}$ & $39,00 \pm 0,38 \mathrm{Aa}^{2}$ & 0,74 \\
\hline TR60* & $39,16 \pm 0,27 \mathrm{Aa}^{2}$ & $39,08 \pm 0,34 \mathrm{Aa}^{2}$ & $38,84 \pm 0,30 \mathrm{Ba}^{1}$ & $38,88 \pm 0,34 \mathrm{Aa}^{2}$ & 0,64 \\
\hline TR80* & $39,11 \pm 0,25 \mathrm{Aa}^{2}$ & $39,07 \pm 0,30 \mathrm{Aa}^{2}$ & $38,81 \pm 0,29 \mathrm{Ba}^{1}$ & $38,84 \pm 0,34 \mathrm{Aa}^{2}$ & 0,69 \\
\hline TR100* & $38,94 \pm 0,24 \mathrm{Aa}^{2}$ & $39,12 \pm 0,32 \mathrm{Aa}^{2}$ & $38,83 \pm 0,28 \mathrm{Aa}^{1}$ & $38,80 \pm 0,39 \mathrm{Ba}^{2}$ & 0,60 \\
\hline
\end{tabular}

TR20, TR40, TR60, TR80 e TR100 referem-se, respectivamente, às temperaturas retais aos 20, 40, 60, 80 e 100 minutos após o exercício físico.

Médias na mesma linha, na mesma raça, seguidas de letras maiúsculas distintas e, no mesmo período, seguidas de letras minúsculas distintas diferem entre si $(\mathrm{P}<0,05)$ pelo teste SNK.

Médias na mesma coluna seguidas de números distintos diferem entre si $(\mathrm{P}<0,05)$ pelo teste Dunnet.

Considerando que o teste foi realizado no turno da manhã (das 7 às 10h), quando a TA ainda estava amena e UR alta a intermediária no período seco (Tab. 5), havia possibilidade de perdas de calor por radiação e convecção, visto que a TA estava menor que a temperatura corporal dos animais. Também foi possível a perda de calor por evaporação visto que a UR esteve sempre abaixo de 100\%. Nessas condições climáticas, as perdas de calor foram provavelmente uma resultante dos três mecanismos, sendo que, no período seco, as perdas por evaporação devem ter sido predominantes. Assim, os animais Saanen, que não conseguiram retornar à temperatura inicial em 100 minutos no período seco, demonstraram maior dificuldade de perda de calor por evaporação em relação aos do grupo Azul.

Tabela 5. Médias das variáveis ambientais temperatura ambiente (TA), umidade relativa (UR) e temperatura de globo negro (TGN) e índice de temperatura e umidade (ITU) e índice de globo e umidade (ITGU) nos períodos chuvoso e seco, em diferentes horários, no momento da realização dos testes de Ibéria, Benezra e Rainsby, no município de Teresina, PI

\begin{tabular}{lcccccc}
\hline \multirow{2}{*}{ Variável } & \multicolumn{3}{c}{ Chuvoso } & \multicolumn{3}{c}{ Seco } \\
\cline { 2 - 6 } & 7 & 10 & 14 & 7 & 10 \\
\hline TA & $27,50 \pm 1,29 \mathrm{Ac}$ & $32,37 \pm 1,39 \mathrm{Ab}$ & $34,50 \pm 1,51 \mathrm{Ba}$ & $26,00 \pm 0,71 \mathrm{Bd}$ & $32,37 \pm 1,48 \mathrm{Ac}$ & $36,50 \pm 1,47 \mathrm{Aa}$ \\
UR & $92,00 \pm 0,00 \mathrm{Aa}$ & $75,00 \pm 8,71 \mathrm{Ab}$ & $67,25 \pm 8,95 \mathrm{Ad}$ & $74,00 \pm 11,36 \mathrm{Ba}$ & $47,00 \pm 4,16 \mathrm{Bb}$ & $29,75 \pm 9,71 \mathrm{Bc}$ \\
TGN & $26,63 \pm 0,97 \mathrm{Bc}$ & $31,87 \pm 1,44 \mathrm{Bb}$ & $33,12 \pm 1,35 \mathrm{Ba}$ & $27,25 \pm 0,84 \mathrm{Ad}$ & $33,75 \pm 1,94 \mathrm{Ac}$ & $38,00 \pm 2,14 \mathrm{Ab}$ \\
ITU & $80,44 \pm 2,21 \mathrm{Ac}$ & $85,68 \pm 1,75 \mathrm{Ab}$ & $87,37 \pm 1,08 \mathrm{Aa}$ & $75,87 \pm 2,33 \mathrm{Bc}$ & $80,65 \pm 1,37 \mathrm{Bb}$ & $82,01 \pm 1,57 \mathrm{Ba}$ \\
ITGU & $77,39 \pm 1,38 \mathrm{Ac}$ & $83,09 \pm 1,48 \mathrm{Ab}$ & $84,35 \pm 1,39 \mathrm{Aa}$ & $76,04 \pm 1,48 \mathrm{Bd}$ & $82,09 \pm 2,09 \mathrm{Bc}$ & $82,90 \pm 2,00 \mathrm{Aa}$ \\
\hline
\end{tabular}

Médias seguidas por letras maiúsculas distintas entre períodos na mesma linha e mesmo horário diferem $(\mathrm{P}<0,05)$ pelo teste SNK. Médias seguidas por letras minúsculas distintas dentro do período e horários diferentes, diferem $(\mathrm{P}<0,05)$ pelo teste SNK.

Martins Júnior et al. (2007a) avaliaram, pelo teste de Rainsby, as raças Boer e Anglo-Nubiana, em condições climáticas similares às deste experimento. Tanto no período chuvoso quanto no seco, os animais da raça Boer tiveram médias iniciais de TR mais baixas $(\mathrm{P}<0,05)$, porém após 10 minutos de exercício e após 60 minutos de repouso pós-exercício, as duas raças comportaram-se de modo semelhante, sem retornar aos valores iniciais de TR.

$\mathrm{Na}$ avaliação da evolução da FR após exercício físico (Tab. 6), observou-se que as cabras do grupo Azul tiveram frequência menor $(\mathrm{P}<0,05)$ que as Saanen a partir dos 20 minutos de descanso, no período chuvoso e durante todo o experimento, desde o repouso até os 100 minutos, no período seco. Observou-se, ainda que no período chuvoso, que elas retornaram aos valores de repouso aos $20 \mathrm{~min}$ e, no período seco, aos $40 \mathrm{~min}$, enquanto as Saanen não retornaram até os 100 minutos em nenhum dos períodos estudados. Isso mostra que a adaptação delas foi mais rápida quanto à $\mathrm{FR}$, semelhante ao observado em relação à TR (Tab. 4). 
Tabela 6. Frequência respiratória (movimentos por minuto), para caprinos Saanen e Azul, em acompanhamento ao teste Rainsby, nos períodos chuvoso e seco, no município de Teresina, PI

\begin{tabular}{llcccc}
\multirow{2}{*}{$\begin{array}{l}\text { Situação/ } \\
\text { Tempo }\end{array}$} & \multicolumn{2}{c}{ Chuvoso } & \multicolumn{2}{c}{ Seco } & CV \\
\cline { 2 - 5 } & \multicolumn{1}{c}{ Saanen } & Azul & Saanen & Azul & $(\%)$ \\
\hline Repouso & $62,06 \pm 29,37 \mathrm{Aa}^{2}$ & $46,13 \pm 20,67 \mathrm{Aa}^{2}$ & $59,31 \pm 21,41 \mathrm{Aa}^{2}$ & $20,75 \pm 5,59 \mathrm{Bb}^{2}$ & 36,86 \\
\hline Exercitado & $132,37 \pm 16,90 \mathrm{Aa}^{1}$ & $110,69 \pm 41,91 \mathrm{Aa}^{1}$ & $129,18 \pm 20,79 \mathrm{Aa}^{1}$ & $79,31 \pm 32,56 \mathrm{Bb}^{1}$ & 27,78 \\
\hline FR20* & $106,63 \pm 20,13 \mathrm{Aa}^{1}$ & $67,31 \pm 30,23 \mathrm{Ab}^{2}$ & $104,31 \pm 23,02 \mathrm{Aa}^{1}$ & $40,25 \pm 14,59 \mathrm{Bb}^{1}$ & 30,39 \\
\hline FR40* & $92,50 \pm 19,44 \mathrm{Aa}^{1}$ & $47,75 \pm 21,29 \mathrm{Ab}^{2}$ & $89,87 \pm 21,12 \mathrm{Aa}^{1}$ & $30,00 \pm 10,84 \mathrm{Bb}^{2}$ & 29,73 \\
\hline FR60* & $84,62 \pm 20,34 \mathrm{Aa}^{1}$ & $44,19 \pm 21,86 \mathrm{Ab}^{2}$ & $89,31 \pm 18,35 \mathrm{Aa}^{1}$ & $24,69 \pm 8,65 \mathrm{Bb}^{2}$ & 30,53 \\
\hline FR80* & $81,82 \pm 25,60 \mathrm{Aa}^{1}$ & $44,31 \pm 23,19 \mathrm{Ab}^{2}$ & $93,94 \pm 24,71 \mathrm{Aa}^{1}$ & $26,81 \pm 10,20 \mathrm{Bb}^{2}$ & 31,90 \\
\hline FR100* & $86,94 \pm 28,59 \mathrm{Aa}^{1}$ & $44,81 \pm 25,70 \mathrm{Ab}^{2}$ & $101,06 \pm 19,52 \mathrm{Aa}^{1}$ & $28,63 \pm 8,32 \mathrm{Bb}^{2}$ & 33,88 \\
\hline
\end{tabular}

FR20, FR40, FR60, FR80 e FR100 referem-se, respectivamente, às frequências respiratórias aos 20, 40, 60, 80 e 100 minutos após o exercício físico.

Médias na mesma linha, na mesma raça, seguidas de letras maiúsculas distintas e, no mesmo período, seguidas de letras minúsculas distintas diferem entre si $(\mathrm{P}<0,05)$ pelo teste SNK.

Médias na mesma coluna seguidas de números distintos diferem entre si $(\mathrm{P}<0,05)$ pelo teste Dunnet.

Os resultados de Medeiros et al. (2002) em teste similar, no Rio de Janeiro, em período quente e úmido, mostram que as cabras Saanen retornaram a FR ao valor inicial aos $45 \mathrm{~min}$, enquanto as Anglo-Nubiana o fizeram após $30 \mathrm{~min}$ de repouso após exercício.

A FC das cabras Saanen (Tab. 7) retornou aos valores de repouso aos 40 minutos durante o período chuvoso e aos 60 minutos no período seco $(\mathrm{P}<0,05)$. Na Azul o retorno foi mais rápido, aos 20 minutos, tanto no período chuvoso, quanto no seco. O aumento de FC após 10 minutos de exercício foi semelhante entre as raças, em ambos os períodos. Esses dados provavelmente refletem maior condicionamento físico cardiovascular das cabras azuis em relação às Saanen. Pode ser também consequência da redução mais rápida da TR (Tab. 4), diminuindo, assim, o estresse dos animais.

Tabela 7. Frequência cardíaca (batimentos por minuto), para caprinos Saanen e Azul, em acompanhamento ao teste Rainsby, nos períodos chuvoso e seco, no município de Teresina, PI

\begin{tabular}{lccccc}
\hline \multirow{2}{*}{$\begin{array}{l}\text { Situação/ } \\
\text { Tempo }\end{array}$} & \multicolumn{2}{c}{ Chuvoso } & \multicolumn{2}{c}{ Seco } & CV \\
\cline { 2 - 5 } & Saanen & Azul & Saanen & Azul & $(\%)$ \\
\hline Repouso & $79,44 \pm 14,89 \mathrm{Aa}^{2}$ & $84,44 \pm 21,86 \mathrm{Aa}^{2}$ & $65,75 \pm 7,29 \mathrm{Bb}^{2}$ & $75,06 \pm 17,72 \mathrm{Aa}^{2}$ & 17,95 \\
\hline Exercitado & $114,19 \pm 14,55 \mathrm{Aa}^{1}$ & $120,25 \pm 34,41 \mathrm{Aa}^{1}$ & $90,56 \pm 17,57 \mathrm{Bb}^{1}$ & $106,06 \pm 17,66 \mathrm{Aa}^{1}$ & 12,59 \\
\hline FC20 & $101,06 \pm 13,39 \mathrm{Aa}^{1}$ & $96,19 \pm 24,22 \mathrm{Aa}^{2}$ & $79,94 \pm 12,71 \mathrm{Ba}^{1}$ & $88,25 \pm 15,70 \mathrm{Aa}^{2}$ & 14,85 \\
\hline FC40 & $87,63 \pm 10,98 \mathrm{Aa}^{2}$ & $88,56 \pm 22,48 \mathrm{Aa}^{2}$ & $76,87 \pm 12,34 \mathrm{Ba}^{1}$ & $78,94 \pm 15,82 \mathrm{Aa}^{2}$ & 13,74 \\
\hline FC60 & $86,19 \pm 11,81 \mathrm{Aa}^{2}$ & $84,44 \pm 21,06 \mathrm{Aa}^{2}$ & $74,56 \pm 10,8 \mathrm{Ba}^{2}$ & $75,87 \pm 14,94 \mathrm{Aa}^{2}$ & 13,64 \\
\hline FC80 & $85,94 \pm 11,76 \mathrm{Aa}^{2}$ & $82,00 \pm 20,72 \mathrm{Aa}^{2}$ & $73,25 \pm 6,28 \mathrm{Ba}^{2}$ & $71,19 \pm 11,68 \mathrm{Aa}^{2}$ & 13,04 \\
\hline FC100 & $81,94 \pm 15,16 \mathrm{Aa}^{2}$ & $84,37 \pm 18,52 \mathrm{Aa}^{2}$ & $73,13 \pm 12,09 \mathrm{Aa}^{2}$ & $68,81 \pm 13,62 \mathrm{Ba}^{2}$ & 15,08 \\
\hline
\end{tabular}

FC20, FC40, FC60, FC80 e FC100 referem-se, respectivamente, às frequências cardíacas aos 20, 40, 60, 80 e 100 minutos após o exercício físico.

Médias, na mesma raça, seguidas de letras maiúsculas distintas na mesma linha e, no mesmo período, seguidas de letras minúsculas distintas diferem entre si $(\mathrm{P}<0,05)$ pelo teste $\mathrm{SNK}$.

Médias na mesma coluna seguidas de números distintos diferem entre si $(\mathrm{P}<0,05)$ pelo teste Dunnet.

A TA, em todos os horários avaliados e nos dois períodos, está acima da zona de termoneutralidade (Tab. 5) para a espécie caprina, 5 a $20^{\circ} \mathrm{C}$, segundo Kolb (1984). Apenas no horário de $10 \mathrm{~h}$ não há diferença $(\mathrm{P}>0,05)$ entre a TA dos períodos seco e chuvoso. Em adição, observa-se comportamento inverso entre TA (crescente) e UR (decrescente) nos diferentes horários do dia nos dois períodos, resultado também observado por Costa et al.
(2004) e Santos et al. (2005), no Meio-Norte do Brasil.

A TGN, aferida à sombra, no período seco, resultou em valores mais altos que os do período chuvoso $(\mathrm{P}<0,05)$, em todos os horários, devido à carga térmica radiante mais intensa neste período. Observa-se, ainda, que a TGN mostra comportamento semelhante ao da TA ao longo do dia e não há na literatura valores de 
referência para a zona de conforto térmico relativa à TGN.

Os resultados obtidos para ITGU durante este experimento (Tab. 5) encontram-se todos acima da zona de conforto, de acordo com o National Weather Service of USA, citados por Baeta (1985). Os resultados observados às sete horas foram mais elevados no período chuvoso $(\mathrm{P}<0,05)$, o que pode explicar a maior $\mathrm{FR}$ em repouso neste período para as cabras Azul e o não retorno à FR do repouso para as cabras Saanen.

Não se tem conhecimento da faixa de ITU que representa a zona de conforto para a espécie caprina, porém de acordo com os valores utilizados por Hahn (1985) numa generalização para diferentes espécies animais e por Silva e Turco (2004), em zoneamento bioclimático para caprinos e ovinos no estado da Bahia os animais deste experimento encontram-se em estresse térmico nos dois períodos e em todos os horários do dia (Tab. 5).

\section{CONCLUSÕES}

No ambiente estudado, os caprinos do grupo racial Azul são mais adaptados visto que têm maior capacidade de manter a temperatura retal, com menor frequência respiratória e maior capacidade de dissipação de calor. O ambiente climático da região Meio-Norte é estressante para caprinos e apresenta valores para índice de temperatura e umidade e de globo e umidade acima dos recomendados como não estressantes.

\section{REFERÊNCIAS BIBLIOGRÁFICAS}

ASSOCIAÇÃO DOS CRIADORES DE CAPRINOS E OVINOS DE PETROLINA E REGIÃO - ASCCOPER. Raças. Petrolina, nov 2005. Disponível em <http://www.asccoper.com.br/exibe>. Acessado em: 26 set. 2006.

BAÊTA, F.C. Responses of lactating dairy cows to the combined effects of temperature, humidity and wind velocity in the warm season. 1985. 218f. Tese (Doutorado em Agricultural Engineering-Structures and Environment) - State University of Missouri, Columbia, EUA.

BUFFINGTON, D.E.; COLLASSO-AROCHO, A.; CANTON, G.H. et al. Black globe-humidity index (BGHI) as confort equation for dairy cows. Transaction of the ASABE, v.24, p.711-714, 1981.
COSTA, A.P.R.; MARTINS JUNIOR, L.M.; AZEVEDO, D.M.M.R. et al. Frequência cardíaca de caprinos Boer e Anglo-Nubiana no período seco e chuvoso em Timon, Maranhão. In: CONGRESSO NORDESTINO DE PRODUÇÃO ANIMAL, 3., 2004, Campina Grande. Anais... Campina Grande: SNPA, 2004. CD-ROM.

FERREIRA, F.; PIRES, M.F.A.; MARTINEZ, M.L. et al. Parâmetros físiológicos de bovinos cruzados submetidos ao estresse calórico. Arq. Bras. Med. Vet. Zootec., v.58, p.732-738, 2006.

HAHN, G.L.; PARKHURRST, A.M.;

GAUGHAN, J.B. Compensatory performance in livestock: influence on environmental criteria. In: YOUSEF, M.K. (Ed.). Stress physiology in livestock. Boca Raton: CRC Press, 1985. v.2, p.52-145.

JARDIM, W.R. Criação de caprinos. 11.ed. São Paulo: Nobel. 1987. 240p.

JOHNSON, H.D. Bioclimatology and adaptation of livestoch. Amsterdam: Elsevier, 1987. 279p.

KOLB, E. Fisiologia Veterinária. 4 ed. Rio de Janeiro: Guanabara Koogan. 1984. 612p.

MAGALHÃES, J.A.; TAKIGAWA, R.M.; TOWNSEND, C.R. et al. Tolerância de bovídeos à temperatura e umidade do trópico úmido. Rev. Cient. Prod. Anim., v.2, p.62-167, 2000.

MAIA, A.S.C.; SILVA, R.G.; ANDRADE, P.C. Efeitos da temperatura e da movimentação do ar sobre o isolamento térmico do velo de ovinos em câmara climática. Rev. Bras. Zootec., v.38, p.104-108, 2009.

MARTINS JÚNIOR, L.M.; AZEVEDO, D.M.M.R.; COSTA, A.P.R. et al. Adaptabilidade das raças Boer e Anglo-Nubiana às condições climáticas da Região Meio-Norte do Brasil. Arch. Zootec., v.56, p.103-113, 2007a.

MARTINS JÚNIOR, L.M.; COSTA, A.P.R.; AZEVEDO, D.M.M.R. et al. Respostas fisiológicas de caprinos Boer e Anglo-Nubiana em condições climáticas de Meio-Norte do Brasil. Rev. Caatinga, v.20, p.1-7,2007b.

MEDEIROS, L.F.D.; VIEIRA, D.H.; QUINTANILHA, J.R. et al. Estimativa da tolerância ao calor em caprinos. Rev. Bras. Med. Vet., v.24, p.30-35, 2002. 
MORAIS, D.A.E.F.; MAIA, A.S.; SILVA, R.G. et al. Variação anual de hormônios tireoideanos e características termorreguladoras de vacas leiteiras em ambiente quente. Rev. Bras. Zootec., v.37, p.538-545, 2008.

MÜLLER, P.B. Bioclimatogia aplicada aos animais domésticos. 2.ed. Porto Alegre: Sulina, 1982. 157p.

NEIVA， J.N.M.; TEIXEIRA， M.; TURCO, S.H.N. et al. Efeito do estresse climático sobre os parâmetros produtivos e fisiológicos de ovinos Santa Inês mantidos em confinamento na região litorânea do Nordeste do Brasil. Rev. Bras. Zootec., v.33, p.668-678, 2004.

OLIVEIRA, L.A.; CAMPELO, J.E.G.; AZEVEDO, D.M.M.R. et al. Estudo de respostas fisiológicas de eqüinos Sem Raça Definida e Quarto de Milha às condições climáticas de Teresina, Piauí. Cienc. Anim. Bras., v.9, p.827$838,2008$.

PANT, K.P.; ARRUDA, F.A.V.; FIGUEIREDO, E.A.P.; Role of gota colour in body heat regulation among gotas and hairy sheep in tropics. Pesq. Agropec. Bras., v.20, p.717-726, 1985.

SANTOS, F.C.B.; SOUSA, B.B.; ALFARO, C.E. et al. Adaptabilidade de caprinos exóticos e naturalizados ao clima semi-árido do Nordeste Brasileiro. Cienc. Agrotec., v.29, p.142 -149, 2005.

SANTOS, F.B.; ACOSTA, A.A.A.; SOUZA, B.B. et al. Avaliação do comportamento fisiológico de caprinos exóticos (Boer e AngloNubiana) e naturalizados (Moxotó e Pardo Sertanejo) sob as condições de clima semi-árido. In: SIMPÓSIO INTERNACIONAL SOBRE
CAPRINOS E OVINOS DE CORTE, 2., 2003, João Pessoa. Anais... João Pessoa: SIMCORTE, 2003. CD-ROM.

SILVA, R.G.; MORAIS, D.A.E.F.; GUILHERMINO, M.M. Evaluation of thermal stress indexes for dairy cows in tropical regions. Rev. Bras. Zootec., v.36, supl., p.1192-1198, 2007.

SILVA, T.G.F.; TURCO, S.H.N. Zoneamento bioclimático de caprinos e ovinos no estado da Bahia. In: REUNIÃO ANUAL DA SOCIEDADE BRASILEIRA DE ZOOTECNIA, 41., 2004, Campo Grande. Anais... Campo Grande: SBZ, 2004. CD-ROM.

SOUZA, B.B.; SILVA, R.M.N.; MARINHO, M.L. et al. Parâmetros fisiológicos e índice de tolerância ao calor de bovinos da raça Sindi no Semi-Árido paraibano. Cienc. Agrotec., v.31, p.883-888, 2007.

SOUZA, B.B.; SOUZA, E.D.; CEZAR, M.F. et al. Temperatura superficial e índice de tolerância ao calor de caprinos de diferentes grupos raciais no Semi-Árido nordestino. Cienc. Agrotec., v.32, p.275-280, 2008a.

SOUZA, B.B.; SOUZA, E.D.; SILVA, R.M.N. et al. Respostas fisiológicas de caprinos de diferentes grupos genéticos no Semi-Árido paraibano. Cienc. Agrotec., v.32, p.314-320, 2008b.

TURCO, S.H.N; ARAÚJO, G.G.L.; BADE, P.L. et al. Respostas fisiológicas de caprinos e ovinos em confinamento a céu aberto, nas condições climáticas do semi-árido nordestino. In: REUNIÃO DA SOCIEDADE BRASILEIRA DE ZOOTECNIA, 41., 2004, Campo Grande. Anais...Campo Grande: SBZ, 2004. CD-ROM. 